\title{
Un cadavre de la Psychanalyse Québécoise. Reflexions sur l'effacement du Père Noël Mailloux
}

\section{A Corpse in the Quebec's Psychoanalysis. Reflections on the erasure of Father Noël Mailloux}

Michel Peterson ${ }^{1}$

Submetido em 4 de abril e aprovado em 14 de abril de 2017.

Résumé: Le Père Noël Mailloux est une des grandes figures intellectuelles du Québec. Il est entre autres le fondateur du département de psychologie de l'université de Montréal et l'instigateur de la psychanalyse au Québec. Cet article présente d'abord les moments clés de son parcours ainsi que les enjeux fondamentaux de sa pratique de transmission au carrefour des sciences humaines (anthropologie, criminologie, etc.). Il présente ensuite son rôle dans la naissance de la psychanalyse québécoise pour ensuite dégager quelques pistes de réflexion autour de l'effacement de sa présence dans la mémoire collective.

Mots clés: Histoire de la psychanalyse québécoise. Institutions. Société psychanalytique de Montréal. Archives. Biographie. Refoulement.

\begin{abstract}
Father Noël Mailloux is one of the major intellectual figures of Quebec. He is, among others, the founder of the department of psychology of the University of Montreal and the instigator of psychoanalysis in Quebec. This paper first presents the key moments of his career as well as the fundamental issues in his practice of transmission to the crossroads of human sciences (anthropology, criminology, etc.). It then presents his role in the birth of the psychoanalysis from in Quebec to then indicate some lines of thought around the disappearance of his presence in the collective memory.
\end{abstract}

Keywords: The history of Quebec psychoanalysis. Institutions. Montreal Psychoanalytic Society. Archives. Biography. Repression.

\section{«Qui se souvient de ce dont 'Je me souviens' doit nous faire souvenir?» Jean Larose, Le mythe de Nelligan.}

Parmi les qualités d'un bon centre au hockey, il y celles d'être un bon passeur pour ses ailiers et de savoir se placer devant le gardien de but adverse pour obstruer sa vue, nuire à son travail et, qui sait?, espérer qu'une rondelle lancée par un coéquipier dévie et trouve le fond du filet. J'ignore s'il pratiqua notre sport national en soutane, mais le fondateur de la psychologie et de la psychanalyse québécoises, le Père Noël Mailloux 
(1909-1997), dominicain de son état, fut l'un de ces passeurs légendaires, dont on remet indéfiniment l'entrée au Temple de la renommée auquel il appartient pourtant, avec les Marguerite Bourgeoys, Marie Victorin, Thérèse Casgrain, Georges-Henri Lévesque, Léa Roback, Armand Frappier, Hans Selye et Fernand Dumont à ce groupe de créateurs et de créatrices d'institutions. Le Révérend Père Mailloux joua pour sa part un rôle crucial dans la constitution des champs de la psychologie, de la psychanalyse et de la criminologie ${ }^{2}$. En ce qui a trait à la psychologie, son importation par « [le grand médiateur» put s'appuyer sur le désir du Père Ceslas-M. Forest, doyen dans les années 30 de la Faculté de philosophie de l'Université de Montréal, désir qui résulta en la création en 1942 de l'Institut de psychologie ${ }^{3}$.

\section{Le fondateur d'institutions}

Nous sommes en 1942 - le Refus global sera publié six ans plus tard. Un jeune et brillant polyglotte revient d'Allemagne et d'Italie. Il a déjà rédigé ses thèses de philosophie et de théologie, et a été ordonné prêtre en 37, dans une église du Corso. Après avoir séjourné à Rome au moment de la montée du fascisme, il fait en 1939 un stage au Son State Hospital, à Cincinnati, où il écoute des psychotiques, avant de devenir professeur de psychologie à l'université d'Ottawa, où il organise un congrès sur l'hygiène mentale et l'éducation.

En 1941, l'université de Montréal, qui souhaite mettre sur pied une école de formation des éducateurs de collège classique, sollicite les services du Père Mailloux en tant qu'enseignant et directeur, ce qu'il accepte avec enthousiasme. Un an plus tard, il fonde le premier département de psychologie du Canada, avant même que ne soit constitué le Allan Memorial Institute. Celui-ci est placé sous la direction du Dr Donald Ewen Cameron, connu pour ses expérimentations financées par la CIA et qui utilise comme cobayes des orphelins de Duplessis. Afin de garantir la crédibilité du nouvel établissement, Mailloux, astucieux, manœuvre pour s'assurer le soutien d'une autorité. Daniel Lagache est approché pour faire partie du corps professoral, mais ce dernier, tout comme Rudolph Loewenstein, l'analyste de Lacan, et John Leuba eux aussi consultés, recommande Théo Chentrier, membre de la Société psychanalytique 
de Paris. Spécialiste en enfance inadaptée, il est expert en délinquance juvénile et juge assesseur auprès du Tribunal de la Seine pour enfants à Paris. D'autres personnes de renom acceptent de cautionner l'«opération» en témoignant de sa valeur scientifique, professionnelle et morale ${ }^{4}$ Le 2 juillet 1948, Beatrice R. Hayes, secrétaire de la Lady Davis Foundation, informe Monseigneur Olivier Maurault, alors recteur de l'Université de Montréal, qu'elle octroie à Chentrier une bourse de 10,000\$ plus les frais de voyage. Il est entendu qu'il sera engagé pour une période de 3 ans, à partir du 1er septembre 1948, à titre de professeur à temps partiel ( 4 cours et 2 séminaires +1 heure de clinique par jour) et qu'il s'occupera de la direction des recherches psychopédagogiques. Il pourra bénéficier d'un versement de $2500 \$$ à son arrivée et de $2500 \$$ chaque année, son salaire annuel étant fixé à 3333.33\$, payable mensuellement ; c'est l'université de Montréal qui défraie les $833.33 \$$ manquants. En fait, Chentrier restera à l'école d'éducateurs jusqu'à sa mort, en juillet 1965, et y deviendra un véritable mentor. Lecteur assidu de Montaigne, Cervantès, sainte Thérèse d'Avila, Racine, Voltaire et Defoe, il publie de très nombreux articles et devient rapidement une présence radiophonique quotidienne, pendant plus d'une décennie, de 1952 à 1964; il est d'abord accompagné par Louise Simard, puis par l'animatrice Lizette Gervais et enfin par Françoise Faucher. Son émission, Psychologie de la vie quotidienne (qui évoque le titre de l'ouvrage de Freud, Psychopathologie de la vie quotidienne), remporte un tel succès que le courrier qu'il reçoit totalise plus de 30,000 lettres par année! En outre, Chentrier fait partie du premier noyau du Mouvement laïque de langue française qui rassemble entre autres des intellectuels comme Jacques Godbout, Jean Le Moyne (ces deux derniers ayant joué le rôle de conseillers auprès de son exécutif), Marcel Rioux, Marcel Dubé, Robert Élie et Solange Chaput-Roland (TESSIER, 2008, p. 45 et sq.). Ainsi, invité au départ par Noël Mailloux, Chentrier va participer de manière significative à la vie du Québec.

Quant au Père Mailloux, alors qu'il aurait pu facilement couler des jours tranquilles à l'ombre de son couvent, il voit toujours plus grand. En 1943, invité par le Conseil des œuvres du diocèse de Montréal et la Fédération des œuvres de charité à prendre en charge les enfants difficiles - c'est-à-dire ceux qui «embêtent» tout le monde, violemment frappés qu'ils sont par les contrecoups de la crise économique que subissent 
leurs parents -, il fonde le Centre d'orientation, qui devient un lieu de formation pour les étudiants de l'Institut de psychologie ${ }^{5}$. Il s'attelle alors à d'importants travaux sur la délinquance juvénile, au point que l'on a pu parler de lui comme du «premier grand 'pionnier fondateur'» de la criminologie québécoise (NORMANDEAU, 1987, p. 68). Le prix de la Société de criminologie du Québec porte d'ailleurs son nom, tout comme l'un des quatre prix de l'Ordre des psychologues.

Mailloux est un One Man Show déterminé qui connaît cependant l'importance du travail en réseau pour aider les jeunes aux prises avec des difficultés de subjectivation. C'est pourquoi il accorde une importance toute particulière aux échanges entre les psychologues du Centre et les éducateurs de Boscoville, ce qui conduira à la création de l'École de psychoéducation de l'Université de Montréal en 1972. Bref, il demeure, avec Bruno Cormier, l'abbé Marc Lecavalier et Denis Szabo, l'un maillons essentiels de l'institutionnalisation de la criminologie et de la psychoéducation ${ }^{6}$.

Mais comme si cela ne suffisait pas, il, vrai bâtisseur, ne s'arrête pas là. Non seulement il met sur pied, en 1946, la Revue de psychologie, puis Contributions à l'étude des sciences de l'homme, puis organise aussi, en 1950, un centre de documentation qui s'appellera bientôt le Centre de recherche en relations humaines dont le modèle à l'époque est Harvard. On y a développé le concept de «relations humaines» qui s'articule en fonction d'un certain nombre de vecteurs - par exemple psychologie, psychologie sociale, sociologie, anthropologie et criminologie. On peut dire que Mailloux fait cohabiter ces vecteurs et c'est Philippe Garigue, anciennement étudiant du London School of Economics, qui reprendra le modèle et l'étendra au moment où il deviendra professeur d'anthropologie familiale à McGill et recrutera des gens formés au Centre de recherche ce que le Père Mailloux n'appréciera guère, d'autant qu'il était plutôt possessif.

\section{La théorie et la pratique}

Plusieurs veines théoriques et cliniques se croisent chez le Père Mailloux. Elles conservent une certaine actualité, pour ne pas dire une actualité certaine, au sens de la pertinence des questions qu'elles posent en regard de la révolution molaire qui déterritorialise aujourd'hui tous les sujets et tous leurs modes d'appréhension du monde. On 
peut dire que de son lieu d'action clinique et politique, où se croisent la foi, la scolastique, la science et la peste - c'est-à-dire Dieu, saint Thomas, la psychologie expérimentale et Freud-, il est d'emblée branché, comme Borduas, sur les flux, les devenirs et les intensités, que Félix Guattari désignait avec précision comme transversalités. Au lieu de cantonner le traitement des délinquants dans une idéologie répressive et conservatrice, Mailloux s'interroge sur les effets comportementaux et psychiques de la course à l'excitation et à la jouissance que promouvait déjà le capitalisme des années 50; des effets qui se sont aggravés depuis que les acteurs financiers, devenus absolument souverains, sont entrés en guerre contre les États de droit et la démocratie, contre la vie. Plutôt que de surveiller et punir, il cherche à penser, dans son travail de fondation et de culture, ce qui est en jeu pour le sujet lorsqu'il est en détresse.

En fait, Mailloux, délinquant parmi les siens, tente de se frayer un chemin vers une question - fort bien formulée par Ilse Barande, une émule de Ferenczi - qui concerne la psychanalyse et l'anthropologie, la sociologie, la science politique et l'économie: «L'appétit d'excitation, la course à l'excitation seraient-ils l'origine et le secret tant de l'aventure de l'hominisation que de la perversion - perversité qui va de pair avec elle? Seraient-ce là les caractéristiques de notre espèce, de son orientation vers un parcours toujours à l'affût, en quête d'obstacles nouveaux lorsque les plus vitaux et les plus urgents ont été maîtrisés?» Voilà une question absolument fondamentale pour penser notre époque et qui, tout en tenant compte de la barbarie de notre situation politique actuelle, la dépasse et plonge dans les couches géologiques de l'espèce humaine. C'est cette question qu'a reprise récemment Barande qui la ramène, ce n'est pas un hasard, au potlatch, à l'économie de la dilapidation théorisée par Bataille sous les termes de la dépense pure. Reprenant la spéculation de Freud à propos de la pulsion de mort, elle précise: «[...] tout se passe comme si cet appétit vital, la course qui s'ensuit, étaient chez l'être humain les témoins d'un en-deçà, d'une fringale sui generis, d'une frénésie du fonctionnement excédant l'aspiration à la réduction des tensions, pour l'engager dans la réalisation d'un maximum de possibilités, fussent-elles extravagantes» (BARANDE, p. 189-190). En d'autres termes - et pour aller au plus urgent-, Mailloux conçoit que le traitement 
des enfants «difficiles» et leur «psychopathologie» passe par une interrogation sur l'ontogenèse et la phylogenèse.

D'où la nécessité d'en venir à... Freud, ce que Mailloux aura fait très tôt, dès le milieu des années 30, en passant par La méthode psychanalytique et la doctrine freudienne, de Roland Dalbiez. Car cette histoire d'excitation, n'est-ce pas dans une tension avec la conscience morale et dans le développement du sentiment de culpabilité lié aux grands renoncements pulsionnels que sont l'interdit de l'inceste et l'œdipe qu'il faut l'aborder? Voilà en tout cas ce que ne cessera d'enseigner le Père Mailloux en appuyant une partie de son enseignement universitaire sur les écrits d'Anne Freud et d'Otto Fenichel, puis sur la seconde topique freudienne, comme on le verra jusque dans le séminaire qu'il dispensera aux Éducatrices de l'École Ste-Hélène en 1982, intitulé Le surmoi et le sens de la vie. C'est le Freud héritier de Luther et de Calvin que retient Mailloux. À ses yeux, rien de rousseauiste : la bonté originelle est un mythe et il va bien falloir assumer que le petit d'homme, ce jouissif «pervers polymorphe», se présente en fait au monde comme «ontologiquement» immoral.

On aura compris qu'il serait intéressant lire Mailloux avec Malaise dans la culture et L'avenir d'une illusion afin d'entendre comment s'articulent la demande économique d'excitation perpétuelle (qui provoque des pathologies qu'on appelle aujourd'hui dépression, boulimie, anorexie, hyperactivité, états-limites, etc.), le penchant de l'homme à l'agression et le sentiment de culpabilité qu'il provoque nécessairement, même s'il est de plus en plus puissamment refoulé.

\section{L'auto-analyse du Père Mailloux}

On peut se demander quelles questions et quelle souffrance auront orienté le Père Mailloux vers de telles interrogations. Ce qui l'agita? Only God knows... On sait qu'il ne se prêta pas à une analyse personnelle, faute de temps, aurait-il affirmé7. Mais que signifie «ne pas avoir le temps», avoir le temps, du temps? Vaste question, surtout s'agissant d'un homme qui passa bel et bien sa vie à donner son temps, à inscrire la psychanalyse dans une histoire personnelle et sociale du don, dans des modes d'échange et de circulation tablant sur le pulsionnel. Si l'histoire sociale du don relève évidemment 
du développement des idéologies religieuses qui motivèrent entre autres l'organisation de l'éducation et des soins de santé durant une longue période de notre histoire, le rapport singulier de Mailloux avec le don, avec sa gratuité, introduisait, à n'en pas douter, par le jeu du transfert, une inquiétance massive au coeur du rapport entre le donateur et les donataires, que ces derniers soient des étudiants, des intervenants ou des délinquants.

Autre question fondamentale: procéda-t-il alors à une auto-analyse (au sens où l'on a pu en parler au sujet de Freud) Si tel est le cas, qui furent ses supports transférentiels, quelle aura été l'adresse de son dire, quel Autre aura-t-il élu comme mandataire de ses fantasmes? Qui auront été ses relais, ses maîtres placés par le transfert en position d'analystes? Faudrait-il penser que le Père Mailloux, aux prises avec un impossible radicalement ouvert par son appel à l'Autre sous la figure de Dieu, en serait venu à recourir à la psychanalyse comme discipline du trouble introduit en tout être par le sexuel et l'infantile? Il est en tout cas possible d'avancer que religion et psychanalyse ne cessèrent de se décentrer en lui chacune par rapport à l'autre. Explorant ce nouage problématique et productif, Jean Bossé parlait de «compromis» $(1984, \text { p. } 51)^{8}$, ce qui se traduit à la lettre par symptôme.

\section{Le refoulement d'un effacement}

Dans l'état actuel de la recherche historique en psychanalyse au Québec - quasi inexistante, il faut le dire -, il serait tout simplement présomptueux et intellectuellement malhonnête d'aller plus loin et de proposer quelque hypothèse que ce soit. Mailloux fut certes un maître qui aura suscité chez ses ouailles, de par sa personnalité passionnée et rigoureuse, des transferts imaginaires sur lui comme supposé détenteur d'une vérité en plus d'avoir engendré de réels transferts de travail chez plus d'un. C'est d'ailleurs à ce double-titre qu'il aura permis une véritable transmission de la psychanalyse québécoise, d'où l'importance de penser le refoulement acharné de sa présence parmi nous. Ses propres filiations sont multiples (européennes et états-uniennes), mais c'est un père peu ou prou reconnu par ses enfants, pourtant nombreux. Le fait qu'il ait porté la robe a-t-il joué? Certainement, mais pas de manière négative, d'autant plus que, comme le soulignait Yvan Lamonde, notre psychanalyse nationale a pu s'inscrire dans le champ 
culturel et intellectuel québécois en obtenant ses trois visas: d'abord religieux (ce qui pose évidemment le problème de la laïcisation de la psychanalyse), puis académique (par le biais de la dialectique entre le thomisme et la psychologie expérimentale) et enfin médical (1984). À partir de cette séquence, mon hypothèse est que les trois stades se sont remplacés l'un l'autre de sorte que le premier (le religieux) s'est trouvé refoulé.

Reste que la prudence est de mise et qu'il ne faut évidemment pas confondre l'histoire de notre psychanalyse avec celle de nos institutions universitaires, médicales et psychanalytiques (PANNACIO, p. 6). Dans les faits, la psychanalyse québécoise qui existe bel et bien en tant que telle dans sa spécificité - s'est au départ constituée sur un ajournement en laissant dans l'ombre une de ses souffrances originaires les plus douloureuses, à savoir le meurtre de son père. C'est ainsi qu'au moment de la fondation, en 1963, de la Canadian Psychoanalytic Society - elle-même héritière du Montreal Psychoanalytic Club, qui se réunit de 1946 à 1952, et compta parmi ses membres, outre le Père Mailloux, un autre fondateur, le Dr Miguel Prados, réfugié antifranquiste, lui aussi autoanalysé -, le rappel historique qui fut présenté alors ne mentionnait ni Mailloux, ni l'Institut de psychologie! L'histoire que se fabrique de ses origines la Société canadienne de psychanalyse mit d'ailleurs en relief la rivalité entre l'Université Mc Gill et l'Institut de psychanalyse au sujet du contrôle de la formation, laquelle recoupait évidemment la dichotomie linguistique (PANNACIO, 71 et 165; PARKIN, 1987; VIGNEAULT, 1993). Filiation paradoxale, s'il en est, que celle qui érige en effaçant, en tentant d'effacer jusqu'à l'effacement même, sans compter que reste toujours un reste qui revient. Dans cette perspective, le propre de la psychanalyse québécoise ne serait-il pas de mettre en scène la pulsion d'agression et de destruction qui préside à toute archive, qui traverse toute cendre, toute mémoire lorsqu'elle met toute sa force à oublier, à ruiner ses traces? Comme quoi son défaut serait on ne peut plus pédagogique.

Bref, la psychanalyse québécoise semble vouée, comme la société de laquelle elle a surgi, à l'effacement des traces et au refus d'inscrire des filiations. Violence originaire qui explique peut-être pourquoi nous ne possédons pas encore de véritable histoire de notre psychanalyse, à part quelques articles, l'alibi habituellement avancé étant la trop grande proximité des événements. Quant à moi, je dirai que la question est ailleurs et 
pourrait se formuler comme suit: comment, au fond, remettre au travail cet originaire qui se donnerait entre autres dans l'archive de la psychanalyse québécoise, si l'on assumait le risque de la constituer? Quand on tente, comme je l'ai fait avec la collection «Voix psychanalytiques», que j'ai fondée et dirigée, d'établir le corpus fondamental d'une discipline et que la collaboration, surtout si on ne bénéficie pas des fonds universitaires, se fait indéfiniment attendre de la part de bien des intéressés, on finit moins par s'épuiser que par revenir à la question que posait jadis Serge Cantin à Jean Bouthillette: « Les Québécois sont-ils même en mesure d'en [la Conquête] rappeler le souvenir à leur mémoire, eux que l'on a ou qui se sont délestés de leur conscience historique?» (p. 45) Pas étonnant qu'il n'y ait que trois fonds d'archives de psychanalyse au Québec, à savoir ceux du Père Mailloux (sur lequel veillent les Dominicains, même si quelques documents dorment à l'université de Montréal), de Julien Bigras (par lui constitué... pour se sauver de l'oubli?) et celui de François Peraldi (que j'ai moi-même établi). C'est peu, mais c'est déjà beaucoup, si l'on considère que la bibliothèque du premier fut dilapidée et que les responsables des Archives de l'Université de Montréal ne crurent pas plus pertinent de constituer celles du second que celles, pour ne prendre que cet exemple, du grand médecin, psychiatre et criminologue Henri Ellenberger, qui publia la célèbre somme intitulée $L a$ découverte de l'inconscient (YANACOPOULO, p. 1).

C'est que nous souffrons, au Québec, d'un mal d'archive, pour reprendre la forte formule de Derrida, qui parle aussi d'un «trouble de l'archive» (p. 140-141). Si l'archivation, en tant qu'institution, est à la fois tournée vers le passé et vers l'avenir, si elle les fait pivoter l'un sur l'autre, l'un dans l'autre; si la psychanalyse, différant en cela de la philologie, engage bel et bien une «nouvelle théorie de l'archive», c'est parce que l'intervention de Freud a laissé en lui sur l'ensemble du savoir humain une impression, au sens où, comme le dit encore Derrida, «on n'a plus le droit ni les moyens de prétendre parler de [l'histoire et de l'historiographie] sans avoir d'avance été marqué, d'une façon ou d'une autre, par cette impression freudienne.» Et cela va encore beaucoup plus loin: «l est impossible et illégitime de le faire sans avoir intégré, bien ou mal, de façon conséquente ou non, en la reconnaissant ou en la déniant, ce qui s'appelle ici l'impression freudienne» (p. 53-54), c'est-à-dire la marque indélébile de la révélation de la pulsion en tout humain 
qui vit. Dénier le Père Mailloux revient ainsi à résister à l'impression freudienne sur lui, revient à perpétuer un refusement de l'après-coup.

Il faut donc se relever les manches car il y a pas mal de boulot, c'est le moins qu’on puisse dire. Dans un exposé donné au séminaire de François Peraldi en mai 1989, Yvan Lamonde dressait déjà un tableau on ne peut plus clair de la situation:

La biographie scientifique des «pères» - Mailloux, Chentrier, Prados, Scott, Lussier, à titre d'exemples - est à faire, tout comme la constitution bibliographique et archivistique de la psychanalyse au Québec. À travers ses institutions - la Faculté de psychologie de l'Université de Montréal (1942), le Montreal Psychoanalytic Club, la Société canadienne de psychanalyse, le Centre de recherches en relations humaines, le Allan Memorial, les Jeudis Laforgue, pour ne nommer que celles-là -, l'institution (et davantage la marge) psychanalytique n'a pas encore accédé à la conscience scientifique. Les filiations individuelles (Chentrier, Charles Odier, Scott - Melanie Klein) et institutionnelle (Montréal-CanadaFrance-Grande-Bretagne-Etats-Unis) demeurent des sentiers historiquement non battus.

Le mystère est d'autant plus grand autour de l'objet qu'on l'a peu nommé.

On pourra certes mieux cerner les conditions de possibilité de la psychanalyse au Québec quand on saura deux ou trois choses d'elle, de ses discours et de ses pratiques. Alors demeurera, au-delà de la trame des traces individuelle et institutionnelle, la question qu'on peut dès maintenant entamer, celle des conditions de possibilité culturelles de ces discours et de ces pratiques, celle d'une tierce dimension à l'objet et au sujet : l'englobant social, culturel $^{9}$.

Depuis lors, soit depuis maintenant plus de 20 ans, la situation n'a pratiquement pas changé, aucune biographie n'a été publiée! Nous n'en savons pas plus sur les Mailloux, Chentrier, Prados et autres (parmi lesquels Mireille Lafortune, Eric Wittkower, Georges Zavitzianos, Gabrielle Clerck) qu'à l'époque ${ }^{10}$. Et quand un ouvrage paraît, à moins qu'il soit publié à Paris («Mon pays, ce n’est pas mon pays...») - y compris et surtout lorsqu'il s'agit d'un Québécois -, il croupit le plus souvent dans les entrepôts jusqu'à ce qu'il soit pilonné, les analystes d'ici ne croyant pas nécessaire de lire ce que leurs collègues publient ici.

Quelques chiffres donneront la mesure de ce désert. On compte au Québec plus de 8300 psychologues, 7896 travailleurs sociaux et thérapeutes conjugaux et familiaux, 1300 criminologues, environ 5200 psychoéducateurs et conseillers et conseillères en 
orientation, cela sans compter les psychothérapeutes et psychanalystes (un grand nombre d'entre eux faisant partie d'un ordre professionnel). Résultat des ventes du $2^{\mathrm{e}}$ volume des œuvres du Père Mailloux en 2009, l'année de parution de l'ouvrage: un exemplaire vendu... Sans compter le pilonnage de plusieurs titres de la collection. Comment comprendre les politiques de dé-subjectivation spécifiques de notre champ social, notre économie libidinale, si nos psychanalystes se lisent pas peu et ne lisent pas les fondateurs, si notre psychanalyse, qui devrait pourtant participer à cette recherche, s'en exclut? Rares furent et son ceux qui se sont risqués à ce travail et se sont risqués sur la scène sociale; mais il y en eut, fort heureusement. C'est qu'il faut prendre l'air de la rue, voir comment ça marche quand le désir est cadenassé par les modes de production capitalistes. À mon sens, les psychanalystes d'ici doivent cesser de répéter ce double geste consistant à adopter une position de maîtres de vérité qui posséderait le savoir de notre culture en gémissant qu'ils ne sont pas entendus. À charge pour les psychanalystes d'affirmer sa place et sa langue en se mouillant et, suivant en cela l'exemple des anciens - Freud et Mailloux - de se manifester dans l'arène publique afin d'en modifier la cartographie libidinale: «La question des conditions de possibilité culturelle de la psychanalyse pratiquée au Québec s'est peut-être muée, en cours de réflexion, en celle des conditions de possibilité culturelle de l'analyse même. Comment l'analytique, le dissociatif, la séparation sontils épistémologiquement et culturellement devenues possibles?» (LAMONDE, p. 21) Cela oblige les analystes à sortir de leur illusoire souveraineté, de leur entre-soi - sans pour autant abandonner leur devoir de réserve - pour prendre en compte, jusqu'au cœur des cures qu'ils dirigent, le champ culturel, pour penser le lien social autrement qu'en rabâchant les mêmes vieilles sempiternelles leçons morales. Ce n'est qu'ainsi qu'on pourra vraiment évaluer comment notre psychanalyse nationale pourrait s'inscrire dans le champ culturel et intellectuel québécois.

Pour le moment en tout cas, aucun travail en profondeur n'a été accompli, d'autant plus qu'aucun argent n'a été investi (sinon celui des éditions Liber) pour permettre que de vraies recherches soient soutenues. C'est dire notre paresse intellectuelle collective. Chacun se défile, s'excuse: pas le temps, trop de clinique. On comprend, en prenant en compte cette fatigue pulsionnelle de notre psychanalyse, que nous n'ayons pas encore 
élaboré de théorie de son institutionnalisation digne de ce nom, alors qu'elles existent en littérature et qu'un imposant travail a été fait en en criminologie, en travail social et dans les sciences appliquées.

$\mathrm{Au}$ tout début d'un texte qui s'appuie sur le commentaire de l'ensemble des contributions publiées dans le numéro de la revue Frayages consacré à la naissance de la psychanalyse à Montréal, François Peraldi dégageait certains faits qui l'avait frappé et qui mettent en lumière les principaux enjeux du rapport symptômal que la psychanalyse québécoise entretient avec l'histoire:

1) un champ psychanalytique non institutionnalisé a pré-existé à Montréal pendant un bon nombre d'années à la formation d'une Société de psychanalyse;

2) ladite Société s'est constituée en partie sur l'exclusion de certains de ceux-là même qui avaient ouvert ce champ;

3) le développement de cette société (sa diversification en plusieurs groupes et instituts et son essaimage à travers le Canada) est fondé sur le refoulement de ses origines (PERALDI, p. 127).

Le découpage proposé par Peraldi inscrit un clivage progressif entre institution et hors-institution, entre centre et périphérie, laquelle opposition comporte toutefois le piège de refouler une topologie autrement plus complexe dans la mesure où quelques-uns traversent plus ou moins constamment les frontières, certains «illégaux» circulant d'un groupe à l'autre afin de se décentrer. Monique Panaccio suggère pour sa part un autre découpage, en quatre périodes, qui met davantage en évidence le désir des protagonistes. À l'époque de la passion, durant les années 40, marquées par le rôle du Montreal Psychoanalytic Club, succède, pendant les années 50 , celle de la demande de reconnaissance auprès des autorités anglaises et états-uniennes. Une fois acquise la légitimité, se fait jour, au cours des années 80 et 90, un sentiment de méfiance envers les institutions, venu tant de l'intérieur que de l'extérieur de l'institution, sentiment qui donne finalement lieu à un mouvement anti-institutionnel, dont le représentant le plus affirmé est justement François Peraldi (PANACCIO, p. 44-45 et sq.). Depuis lors, les groupes hors-institution se sont multipliés, la plupart d'inspiration plus ou moins fortement d'inspiration lacanienne. 


\section{Le nom du cadavre}

En éditant une partie des œuvres du Père Mailloux, il m’est arrivé à plusieurs reprises de penser au géant Beaupré, né en 1881 en Saskatchewan, et mort en 1904 de la tuberculose dans un hôpital de Saint-Louis. C'est que je me posais une simple question : comment commence-t-on sa vie chez nous après la mort?

L'histoire du géant canadien est riche d'enseignement en ce qui a trait à l'effacement. Pauvre comme Job, notre géant ne pouvait se payer le luxe de funérailles. Pour récupérer sa mise, son patron le fit embaumer et l'exposa dans la vitrine d'un magasin. C'est alors que commence la partie fabuleuse de l'histoire. Un inconnu, touché par la grandeur d'Édouard, fait pétrifier son corps pour l'apporter à Montréal. Nonobstant le refus des autorités et un court séjour dans un musée d'anatomie de Saint-Louis, il se retrouve, on ne se sait par quelle magie, dans un musée de la rue Saint-Laurent, où il habite pendant six mois, jusqu'à qu'il soit... perdu! La légende dit que des enfants découvrirent le cadavre du géant au printemps 1907 dans un entrepôt, oublié là par les responsables d'un cirque qui avaient projeter de l'exposer au parc Maisonneuve. C'est alors qu'un médecin, professeur à l'université de Montréal, achète le cadavre pour 25 dollars. Il l'empaille et l'expose, debout, dans une cage de verre, à l'entrée d'un laboratoire, là où, comme le dit la chanson, son gardien passera des nuits à jaser avec son squelette gêné, à l'abri du monde fou. Notre géant doit attendre 1990 pour être libéré et que l'on renvoie ses cendres dans sa famille à Willow Bunch, où on érige une statue en son honneur.

Un cadavre circule depuis bien des années au cœur de la psychanalyse québécoise. Personne ne le garde puisque point de crypte. Mailloux est le nom de ce cadavre, du cadavre de notre psychanalyse, tombé de ses cendres.

Or ce nom n'est pas le seul à être victime de la défaillance historique de notre mémoire, au point que nous ne savons même plus que nous avons oublié. Dans une chronique de mars 2009, Pierre Foglia parlait du dernier hommage rendu à André Langevin, à l'hôtel de ville de Frelishburg et soulignait que la famille des écrivains s'était absentée, seuls Françoise Faucher, Charles Tisseyre et Pierre Filion, s'étant souvenus de cet homme et de son œuvre (FOGLIA, p. A-5). Poussière sur le nom... comme sur celui du père Marie-Alain Couturier (1897-1954). Venu de la Loire et séjournant à plusieurs 
reprises à Montréal de 1940 à 1945, ce dominicain joua un rôle crucial auprès de Borduas, alors qu'ils enseignaient tous deux à l'École du meuble et discutaient de questions relatives à l'art sacré et à l'abstraction. Voilà un autre homme de robe qui compta dans le développement des réflexions qui allaient conduire au Refus global, au point que Borduas, dans Projections libérantes et Gauvreau, dans ses Écrits sur l'art, insistent sur l'importance de sa contribution (SCHARTZWALD, p. 82) ${ }^{11}$.

Ce qui est en jeu dans cet oubli massif de quelques grands noms et contre lequel lutte par exemple d'une manière colossale Victor Lévy Beaulieu (mais il n'est pas complètement seul, fort heureusement), c'est, plus encore que le rejet de l'institution ecclésiastique, le métarécit glorieux de la Révolution tranquille, lequel nous aide à nous voiler la face, à oublier notre veulerie. Un cadavre oublié revient toujours au cœur des ténèbres, lorsque nous dormons du sommeil de l'injuste. Ce pourquoi nous ne devons pas simplement, contre nos gouvernements, redorer le corps mort abandonné dans une grange, réparer l'histoire, mais commencer à la penser avec ses trous, ses lacunes, comprendre si et comment elle fait symptôme de l'histoire intellectuelle et institutionnelle du Québec.

\section{Références}

BARANDE, Ilse. L'appétit d'excitation. Paris: PUF, 2009

BOSSÉ, Jean. L'analyse profane?. Frayages, no 3, 51-55, 1984

BRODEUR, Claude. Parcours d'un psychanalyste. Le cheminement de l'idée. Montréal: Liber, 2008

Bulletin de la Société psychanalytique de Montréal, vol. 4, no 1, automne 1991.

CANTIN, Serge et BOUTILHETTE, Jean. «Lettres sur le Québec». Liberté, no 240, vol. 40-6, p. 24-50, 1988

CLOUTIER, Yvan, Les dominicains et l'import-export: psychanalyse et existentialisme au Québec, Horizons philosphiques, vol. 2, no 1, 91-105, 1991.

DERRIDA, Jacques. Mal d'archive. Paris : Galilée, 1995

FOGLIA, Pierre. Chronique. La Presse, 21 mars, p. A-5, 1995

HAMELIN, Monique. Entretien avec le père Mailloux. LEBOEUF, M.-E. et GAUTHIER, D. (éds.), Penser la criminologie : propos recueillis sur 25 ans de criminologie au Québec, Montréal, Cahiers de l'École de criminologie, Université de Montréal, 17-31, 1986. 
LAMONDE, Yvan. Psychanalyse et topique historique, Frayages, no 3, 15-22, 1984 LOWY, Fred et alii. History and Culture in Canadian Psychoanalysis. Canadian Journal of Psychoanalysis, vol. 16, no 1, p. 74-94, 2008.

MAILlOUX, Noël. Savoirs psychologiques et débats moraux. Choix de textes 2. Montréal: Liber, 2009

MAILLOUX, Noël. Psychologie, psychologie clinique, psychodynamique. Choix de textes 1. Montréal: Liber, 2007

NORMANDEAU, André.La petite histoire de la criminologie au Québec (1960-1985), Revue internationale de criminologie et de police technique, no 1, 67-80, 1987.

PANNACIO, Monique. La psychanalyse au Québec: formation, filiation, transmission. Thèse de doctorat, Université du Québec à Montréal, Département de Psychologie, 2000.

PARKIN, Alan. History of Psychoanalysis in Canada. Toronto: Toronto, 1987. Psychoanalytic Society.

PELLETIER, Pierre. Le psychanalyste et le Bouddha. Itinéraire spirituel. Montréal: Liber, 2008.

PERALDI, François. La marge psychanaytique, Frayages, no 3, p. 127-135, 1984

PERALDI, François. Séminaire 4. L'enseignement de Jacques Lacan. Montréal: Liber (à paraître).

SCHARTZWALD, Robert (2004). «Un apport singulier à l'avénement de la modernité au Québec. Hommage au père Marie-Alain Couturier, O.P., à l'occasion du cinquantième anniversaire de sa mort». In: MICHAUD, Ginette et NARDOUTLAFARGE, Élisabeth, dirs. Constructions de la modernité au Québec, Ginette Michaud et Élisabeth dir., Montréal, Lanctôt, p. 79-125.

SZABO, Denis. Entretiens avec Marcel Fournier. Fondation et fondements de la criminologie. Montréal: Liber, 1998

TESSIER, Nicolas. Le Mouvement laïque de langue française: laïcité et identité québécoise dans les années 60. Mémoire de maîtrise, Département d'histoire, Université de Montréal, 2008

VIGNEAULT, Jacques. Transferts et déplacements. Fondements de la psychanalyse en Amérique du Nord. Trans, no 3, p. 223-237, 1993.

YANACOPOULO, Andrée. Entretien. Liber Bulletin, p. 1, 2009

\section{Notes}

${ }^{1}$ Psychanalyste, professeur visitant au Département de psychologie de l’Université Fédérale de Rio Grande et chercheur à la Chaire Oppenheimer en Droit International Public, Université McGill, Montréal, Canada. profmichelpeterson@gmail.com. 
2 Par souci d'économie, je renvoie sur ce legs à ma présentation du premier volume de ses œuvres, éditées par mes soins: Psychologie, psychologie clinique, psychodynamique. Choix de textes, 1, Montréal, Liber, 2007, p. 7-23. Un second volume est paru. Il s'agit de Savoir psychologique et débats moraux, Choix de textes, 2, Montréal, Liber, 2009.

${ }^{3}$ Concernant les étapes et les figures de proue (le Père Marcel-Marie Desmarais, le Dr Antonio Barbeau, le Dr Miguel Prados, Théo Chantrier, Gregor Zylboorg, etc.) de la constitution de la psychanalyse au Québec, on peut lire Yvan Cloutier, «Les dominicains et l'import-export : psychanalyse et existentialisme au Québec» (1991, en particulier p.97-99).

${ }^{4}$ On peut citer ici entre autres Juliette Boutonnier, co-fondatrice de la Société Française de Psychanalyse et présidente de l'Association Française de Criminologie de 1965 à 1968; Maurice Dubourge, archevêque de Besançon; Robert Chadefaux, Président du Tribunal pour Enfants de la Seine et Conseiller à la Cour d'Appel de Paris; et Monseigneur Simon Delacroix, Préfet de la Maison de Sa Sainteté et Professeur à l'Institut Catholique de Paris.

${ }^{5}$ Ainsi qu'en témoigne par exemple le programme de formation des éducateurs spécialisés (qui inclut le certificat de psycho-pédagogie de l'enfance inadaptée et la licence en éducation spécialisée) tel que répertorié dans les annuaires de l'Institut de psychologie de la Faculté de philosophie de l'université de Montréal. Voir, pour la constitution de ce programme, «Entretien avec le père Mailloux». Propos recueillis par Monique Hamelin (1986).

${ }^{6}$ Pour mémoire: Bruno Cormier (1919-1991) psychanalyste, l'un des signataires du Refus Global - que l'on peut considérer comme un précurseur de Julien Bigras en ce qui a trait à l'articulation du lien entre art et psychanalyse - fonde, en 1955, la Clinique de psychiatrie légale à la Faculté de médecine de l'université McGill, rien de moins!, au moment même où il commence à travailler comme clinicien au pénitencier de Saint-Vincent de Paul. Un maigre numéro du Bulletin de la Société psychanalytique de Montréal (automne 1991) lui a été consacré au moment de sa disparition. Pour sa part, le père Lecavalier, travailleur social et président de l'Association des aumôniers au service des pénitenciers et des prisons, convainc le ministère du Bien-Être social de l'époque de mettre en place des cliniques de diagnostic et d'aide à l'enfance. Denis Szabo, originaire de Hongrie, fonde de son côté l'enseignement de la criminologie à l'université de Montréal. Dans ses entretiens avec Marcel Fournier (1998), il explique à plusieurs reprises le rôle qu'y a tenu Noël Mailloux. ${ }^{7}$ C'est en tout cas la réponse qu'il fournit à Monique Panaccio lorsqu'elle lui posa la question (2000).

${ }^{8}$ Le nouage en question, qui implique nécessairement le politique, est par exemple au coeur d'une lettre datée du 27 juillet 1951 adressée par le Père Mailloux au Père Emmanuel Suarrez, à l'époque Maître Général à Rome de l'Ordre des Frères Prêcheurs, au sujet de la conversion du Dr Gregory Zylboorg au catholicisme et des difficultés que posent ce geste. Le Père Mailloux écrit: «Le Dr Zilboorg [sic] est juif, et fut d'abord marié à une juive dont il eut deux enfants [mariage qui eut lieu en 1919]. Il y a quatre ou cinq ans, cette femme demanda et obtint le divorce devant la Cour des États-Unis. Aussitôt après, le Docteur se mariait de nouveau avec une femme de religion anglicane [le 19 août 1946]. De ce second mariage aussi deux enfants sont nés. Cette femme appartenant à la Haute Église, pratique dévotement sa religion, et j'ai l'impression très nette qu'elle ne tarderait pas à suivre son mari dans la Foi catholique, advenant le baptême de ce dernier. [...] Nous nous sommes donc demandé si le privilège paulin ne pourrait pas être appliqué au premier mariage, ouvrant ainsi la possibilité d'obtenir les dispenses nécessaires en vue de la validation du second. [...] Enfin, pour de nombreuses raisons, il me paraît souhaitable que la conversion du Dr Zilboorg [sic] ainsi ques on baptême puissent s'accomplir d'une façon plutôt discrète. Cet homme ayant été jadis le secrétaire de Kerensky, a été mêlé à la politique active. D'autre part, ses sympathies manifestes à l'égard de l'Église catholique ont entraîné la réprobation d'ennemis puissants de l'Église.» Zylboorg sera effectivement baptisé le 2 octobre 1954 (comme en témoigne une lettre, datée du 30 septembre 1954, du Père Mailloux à Mgr Olivier Maurault, alors recteur de l'université de Montréal). Le nouage religion-politique est clairement posé par l'intéressé dans une lettre au Très Saint-Père: «Le problème des religions et du christianisme commença à m'intéresser alors que j'avais 19 ou 20 ans. Mais c'est surtout après la révolution bolchéviste que mes préoccupations religieuses se firent de plus en plus sentir» (31 janvier 1953).

${ }^{9}$ Cet exposé fait partie du 4e et dernier tome du Séminaire de François Peraldi, à paraittre chez Liber.

${ }^{10}$ Dans la collection «Voix psychanalytiques», nous tentons d'entreprendre ce travail et avons jusqu'à ce jour publié deux ouvrages en ce sens: ceux de Claude Brodeur (2008) et de Pierre Pelletier.

${ }^{11}$ Robert Schwartzwald mentionne également l'intérêt du père Couturier pour le modernisme artistique, au point d'avoir collaboré avec Le Corbusier, Léger et Matisse. 